\section{Case Reports in Oncology}

\title{
Significance of Earlier Initiation of Chemotherapy for Lung Cancer Complicated with Primary or Secondary Nephrotic Syndrome following Its Appropriate Differential Diagnosis
}

Shuji Ota ${ }^{a}$ Yoshihide Fujigaki $^{b} \quad$ Yoshifuru Tamura $^{b} \quad$ Kenichiro Kojima $^{c}$ Ryosuke Ochiai ${ }^{a}$ Terunobu Haruyama ${ }^{a}$ Masashi Ishihara ${ }^{a}$ Maika Natsume ${ }^{a}$ Yoko Fukasawa $^{a}$ Takahiko Sakamoto $^{a}$ Shigeru Tanzawa ${ }^{a}$ Ryo Usui ${ }^{a}$ Takeshi Honda ${ }^{a}$ Yasuko Ichikawa ${ }^{a}$ Kiyotaka Watanabe ${ }^{a}$ Nobuhiko Seki ${ }^{a}$

aDivision of Medical Oncology, Department of Internal Medicine, Teikyo University School of Medicine, Tokyo, Japan; ${ }^{b}$ Department of Internal Medicine, Teikyo University School of Medicine, Tokyo, Japan; 'Department of Nephrology, Ageo Central General Hospital, Ageo, Japan

\section{Keywords}

Lung cancer $\cdot$ Nephrotic syndrome $\cdot$ Chemotherapy

\begin{abstract}
We encountered a case of primary lung cancer complicated with membranous nephropathy as primary nephrotic syndrome. Because treatment approaches vary greatly for primary and secondary nephrotic syndrome, a renal biopsy was performed for diagnosis. Much time was required to make a definitive diagnosis of primary nephrotic syndrome, as opposed to paraneoplastic nephrotic syndrome. Consequently, the subsequent chemotherapy was ineffective and caused significant toxicity due to reduced performance status (PS) and progression of
\end{abstract}


hypoalbuminemia. Therefore, it is imperative that a diagnosis be made and treatment be initiated without delay before PS declines and hypoalbuminemia progresses.

\author{
(C) 2019 The Author(s) \\ Published by S. Karger AG, Basel
}

\title{
Introduction
}

Nephrotic syndrome is known to occur as a part of paraneoplastic syndromes that complicate malignant cancer [1]. Correct diagnosis of nephrotic syndrome is extremely important, because treatment approaches for primary versus paraneoplastic nephrotic syndrome are very different. However, there is a risk that making a definitive diagnosis may require significant amount of time, because renal biopsy is usually performed. In this report, we discuss the important issues in the chemotherapy of primary lung cancer complicated with nephrotic syndrome.

\section{Case Report}

The patient was a 75-year-old male who presented with chief complaints of edema in lower extremities slowly progressing since three weeks prior, and a 4-kg weight gain. He had a 40-pack-year history of smoking. His PS was 1 at the time of admission. Pitting edema was observed in both lower legs. Laboratory data at admission included the following values: urine protein, $7.1 \mathrm{~g} / \mathrm{day}$; serum albumin, $2.2 \mathrm{~g} / \mathrm{dL}$; serum total protein, $5.1 \mathrm{~g} / \mathrm{dL}$; and serum total cholesterol, $372 \mathrm{mg} / \mathrm{dL}$. Based on these findings, a diagnosis of nephrotic syndrome was made.

On the other hand, in the imaging tests at the time of admission, a mass was observed in the right lower lung field (Fig. 1a, b). A biopsy of the mass was performed, and a diagnosis of stage IV squamous cell lung cancer (cT3N2M1b) was made. The tumor marker, squamous cell carcinoma antigen (SCC), was elevated at $17.0 \mathrm{ng} / \mathrm{mL}$ (normal: $<1.5 \mathrm{ng} / \mathrm{mL}$ ). At this point, correct diagnosis of nephrotic syndrome was required because treatment approaches for primary versus paraneoplastic nephrotic syndrome are very different. Then, histopathological findings of the renal biopsy included mild thickening of the glomerular walls in HE-stained specimens (Fig. 2a) and spikes of the glomerular basement membrane protruding towards the epithelium in PAM-stained specimens (Fig. 2b). Immunofluorescence staining showed granular deposition of IgG on the glomerular walls (Fig. 3a). IgG subclass analysis showed predominant deposits of IgG4 (Fig. 3b), which is consistent with the staining patterns in primary membranous nephropathy. Other possible causes of secondary membranous nephropathy were hepatitis $B$ and hepatitis $C$ virus infections, connective tissue disease, and drug induced disease, which were excluded in this case.

Eventually, a total of 30 days were required for us to determine the cause of the nephrotic syndrome, from admission to initiation of chemotherapy. By the time of chemotherapy initiation, the patient's PS had worsened to 2, with edema in lower extremities due to progressing nephrotic syndrome, and serum albumin had further reduced to $1.1 \mathrm{~g} / \mathrm{dL}$. Initial chemotherapy regimen was the standard carboplatin (AUC 6)/paclitaxel $\left(200 \mathrm{mg} / \mathrm{m}^{2}\right)$ combination therapy. During the first cycle of chemotherapy, side effects included grade 4 neutropenia and grade 4 thrombocytopenia, and the PS worsened to 3 due to grade 2 fatigue. The tumor grew after the first cycle, upon which we had no choice but to provide best supportive care. 
 Oncology}

\section{Discussion}

The incidence of malignancy-associated membranous nephropathy has been reported to be relatively high. A retrospective study evaluated 107 cases of biopsy-proven membranous nephropathy, and 9 cases (8.4\%) were found to be associated with malignancy [2]. A pathogenic mechanism for malignancy-associated nephrotic syndrome has been proposed [3, 4]. In membranous nephropathy, tumor-associated antigens are found in glomerular lesions, suggesting a possibility that the nephropathy is caused by the deposition of immune complexes of tumor-associated antigens and their IgG antibodies. Furthermore, regarding the immunofluorescence staining of IgG subclass, it is shown that IgG4 predominantly deposits on the glomerular walls in primary membranous nephropathy, whereas IgG1 and IgG2 predominantly deposit in secondary membranous nephropathy. In this case, IgG4 deposits were mainly observed, resulting in the diagnosis of primary membranous nephropathy.

Primary nephrotic syndrome is primarily treated with systemic steroid administration. In contrast, priority is given to the treatment of cancer in cases of paraneoplastic nephrotic syndrome, and an improvement in nephrotic syndrome can be obtained by successful treatment of the cancer. Because treatment approaches vary greatly for primary and secondary nephrotic syndrome, a renal biopsy is often performed for its appropriate differential diagnosis although it may spend a lot of time, including consultation with the division of nephrology, application of renal biopsy, addition of the immunofluorescence staining to the biopsy specimens, pathological diagnosis, and clinicopathological conference between the divisions of pathology, nephrology, and medical oncology.

According to the guidelines for timely initiation of chemotherapy, chemotherapy should commence within 3 weeks of the ready for care date in patients with advanced non-small-cell lung cancer on the basis of the statistical analysis that increased time-to-chemotherapy shows negative prognostic impact [5]. This recommendation is supported by the facts that chemotherapy in cancer patients whose original PS worsened over time is associated with increased toxicity and poor prognosis [6-8]. On the other hand, in our patient, we should have taken care that his PS also tends to worsen along with the signs and symptoms of his untreated nephrotic syndrome, including hypoalbuminemia.

Regarding hypoalbuminemia in nephrotic syndrome, reduction in plasma albumin concentration is associated with decrease in plasma protein binding of highly bound drugs, allowing for higher unbound drug levels. Therefore, toxicity of anti-cancer drugs with high plasma albumin binding rate could be especially increased. In fact, multiple studies reported that hypoalbuminemia is a risk factor for febrile neutropenia in cancer chemotherapy [9-11]. Furthermore, although carboplatin and paclitaxel used in our case is generally known as one of the well-tolerated regimens, in cases of hypoalbuminemia, it was reported that even this regimen was associated with more toxicities, including anemia, fatigue, and anorexia, when compared with normal cases [12]. With these contributing factors, studies found that the prognosis of lung cancer complicated with nephrotic syndrome is very poor [13], and that proteinuria in lung cancer can be a prognostic factor [14].

In conclusion, in terms of the chemotherapy-induced toxicity and prognosis, earlier initiation of chemotherapy for lung cancer complicated with primary or secondary nephrotic syndrome following its appropriate differential diagnosis is imperative before PS declines and hypoalbuminemia progresses. 
Ota et al.: Significance of Earlier Initiation of Chemotherapy for Lung Cancer Complicated with Primary or Secondary Nephrotic Syndrome following Its Appropriate Differential Diagnosis

\section{Statement of Ethics}

The authors have no ethical conflicts to disclose.

\section{Disclosure Statement}

The authors have no conflicts of interest to disclose.

\section{References}

1 Lee JC, Yamauchi H, Hopper J Jr. The association of cancer and the nephrotic syndrome. Ann Intern Med. 1966 Jan;64(1):41-51.

2 Burstein DM, Korbet SM, Schwartz MM. Membranous glomerulonephritis and malignancy. Am J Kidney Dis. 1993 Jul;22(1):5-10.

3 Doi T, Mayumi M, Kanatsu K, Suehiro F, Hamashima Y. Distribution of IgG subclasses in membranous nephropathy. Clin Exp Immunol. 1984 Oct;58(1):57-62.

4 Haas M. IgG subclass deposits in glomeruli of lupus and nonlupus membranous nephropathies. Am J Kidney Dis. 1994 Mar;23(3):358-64.

5 Alexander M, Beattie-Manning R, Blum R, Byrne J, Hornby C, Kearny C, et al. Guidelines for timely initiation of chemotherapy: a proposed framework for access to medical oncology and haematology cancer clinics and chemotherapy services. Intern Med J. 2016 Aug;46(8):964-9.

6 Stanley KE. Prognostic factors for survival in patients with inoperable lung cancer. J Natl Cancer Inst. 1980 Jul;65(1):25-32.

7 Albain KS, Crowley JJ, LeBlanc M, Livingston RB. Survival determinants in extensive-stage non-small-cell lung cancer: the Southwest Oncology Group experience. J Clin Oncol. 1991 Sep;9(9):1618-26.

8 Paesmans M, Sculier JP, Libert P, Bureau G, Dabouis G, Thiriaux J, et al.; The European Lung Cancer Working Party. Prognostic factors for survival in advanced non-small-cell lung cancer: univariate and multivariate analyses including recursive partitioning and amalgamation algorithms in 1,052 patients. J Clin Oncol. 1995 May;13(5):1221-30.

9 Kloft C, Wallin J, Henningsson A, Chatelut E, Karlsson MO. Population pharmacokinetic-pharmacodynamic model for neutropenia with patient subgroup identification: comparison across anticancer drugs. Clin Cancer Res. 2006 Sep;12(18):5481-90.

10 Alexandre J, Gross-Goupil M, Falissard B, Nguyen ML, Gornet JM, Misset JL, et al. Evaluation of the nutritional and inflammatory status in cancer patients for the risk assessment of severe haematological toxicity following chemotherapy. Ann Oncol. 2003 Jan;14(1):36-41.

11 Lyman GH, Delgado DJ. Risk and timing of hospitalization for febrile neutropenia in patients receiving CHOP, CHOP-R, or CNOP chemotherapy for intermediate-grade non-Hodgkin lymphoma. Cancer. 2003 Dec;98(11):2402-9.

12 Arrieta O, Michel Ortega RM, Villanueva-Rodríguez G, Serna-Thomé MG, Flores-Estrada D, Diaz-Romero C, et al. Association of nutritional status and serum albumin levels with development of toxicity in patients with advanced non-small cell lung cancer treated with paclitaxel-cisplatin chemotherapy: a prospective study. BMC Cancer. 2010 Feb;10(1):50.

13 Coltharp WH, Lee SM, Miller RF, Averbuch MS. Nephrotic syndrome complicating adenocarcinoma of the lung with resolution after resection. Ann Thorac Surg. 1991 Feb;51(2):308-9.

14 Pedersen LM, Milman N. Prevalence and prognostic significance of proteinuria in patients with lung cancer. Acta Oncol. 1996;35(6):691-5. 


\section{Case Reports in Oncology}

\begin{tabular}{l|l}
\hline Case Rep Oncol 2019;12:53-58 \\
\hline DOI: 10.1159/000493851 & $\begin{array}{l}\text { c) 2019 The Author(s). Published by S. Karger AG, Basel } \\
\text { www.karger.com/cro }\end{array}$
\end{tabular}

Ota et al: Significance of Earlier Initiation of Chemotherapy for Lung Cancer Complicated with Primary or Secondary Nephrotic Syndrome following Its Appropriate Differential Diagnosis
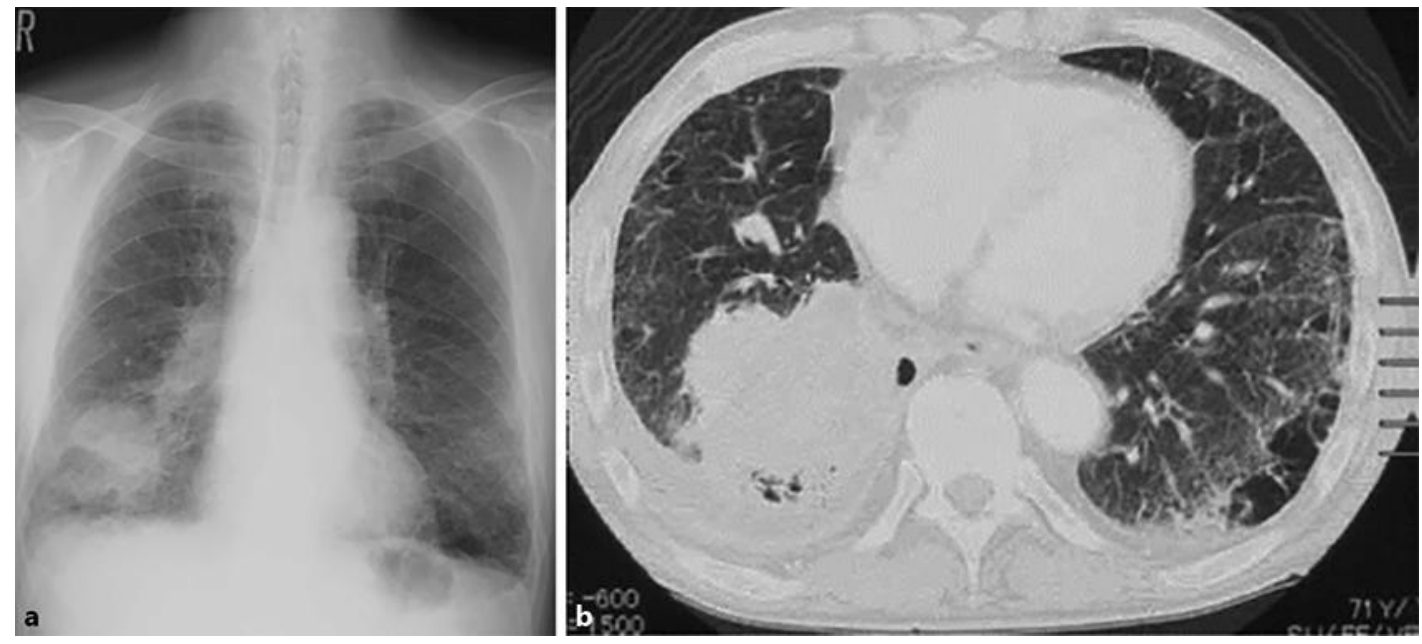

Fig. 1. a: A mass is visible in the right lower lung field. b: A mass of $5 \mathrm{~cm}$ in diameter is visible in right S8.

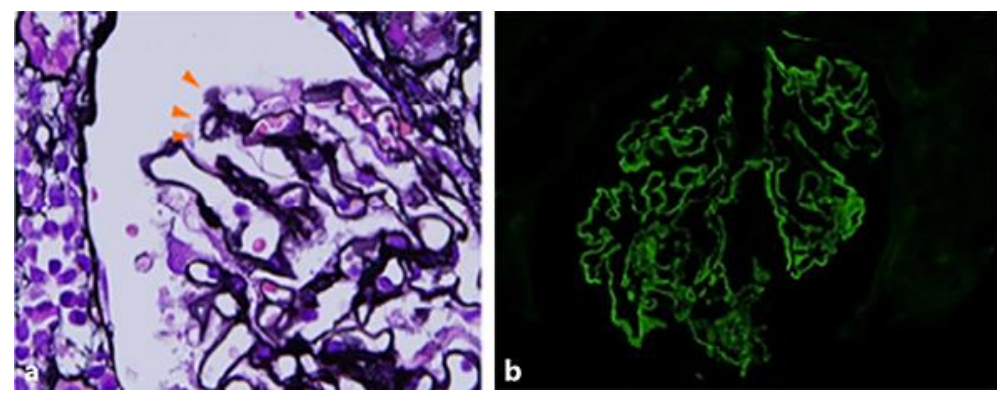

Fig. 2. a: Masson trichrome staining. Mild thickening of the glomerular walls is visible. b: PAM staining. Spikes of the glomerular basement membrane protruding towards the epithelium are visible (arrowheads). 


\section{Case Reports in Oncology}

\begin{tabular}{l|l}
\hline Case Rep Oncol 2019;12:53-58 \\
\hline DOI: 10.1159/000493851 & $\begin{array}{l}\text { (c) } 2019 \text { The Author(s). Published by S. Karger AG, Basel } \\
\text { www.karger.com/cro }\end{array}$
\end{tabular}

Ota et al.: Significance of Earlier Initiation of Chemotherapy for Lung Cancer Complicated with Primary or Secondary Nephrotic Syndrome following Its Appropriate Differential Diagnosis
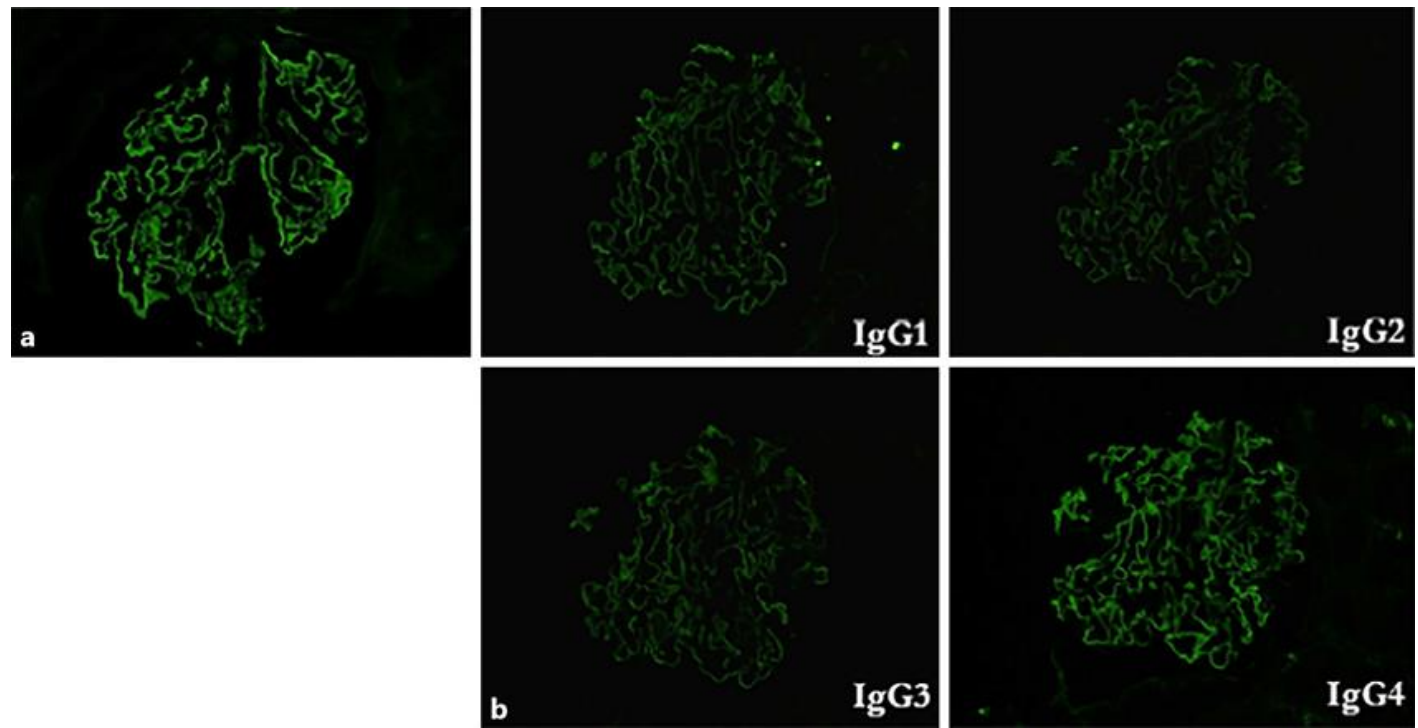

Fig. 3. a: Immunofluorescence staining. Granular deposition of IgG on the glomerular walls is visible. b: Immunofluorescence staining. IgG4 deposits are predominantly visible. 